NASA Technical Memorandum 105282

\title{
A New Approximate Sum Rule for Bulk Alloy Properties
}

Guillermo Bozzolo Analex Corporation

Brook Park, Ohio

and

John Ferrante

Lewis Research Center

Cleveland, Ohio

October 1991 


\title{
A NEW APPROXIMATE SUM RULE FOR BULK ALLOY PROPERTIES
}

\author{
Guillermo Bozzolo \\ Analex Corporation \\ 3001 Aerospace Parkway \\ Brook Park, Ohio 44142-1003 \\ John Ferrante \\ National Aeronautics and Space Administration \\ Lewis Research Center \\ Cleveland, Ohio 44135-3191
}

\begin{abstract}
We introduce a new, approximate sum rule for determining bulk properties of multicomponent systems, in terms of the pure components properties. We apply this expression for the study of lattice parameters, cohesive energies and bulk moduli of binary alloys. The correct experimental trends (i.e., departure from average values) are predicted in all cases.
\end{abstract}


In this paper we introduce a new expression for the direct calculation of equilibrium properties of alloys in terms of the pure components. From this new rule, which can be obtained as a particular case from the method for alloys energetics introduced recently by Bozzolo, Ferrante and $\mathrm{Smith}^{(1)}$, we can simultaneously derive three separate expressions for obtaining the lattice parameter, cohesive energy and bulk modulus of alloys.

Consider an alloy of $\mathrm{N}$ different elements $A_{x_{A}} B_{x_{B}} \ldots$ where $x_{i}$ denotes the concentration of the atomic species $i$. Let $E_{C}(x), a(x)$ and $B(x)$ be the cohesive energy, lattice parameter and bulk modulus, respectively, of the alloy characterized by the concentrations $x:\left(x_{A}, x_{B}, \ldots\right)$. Then, our approximate expression for the binding energy as a function of concentration $x$ and lattice parameter $r, E(x, r)$, in terms of the corresponding expressions for the pure elements is given by

$$
E(x, r)=\sum_{i} x_{i} E_{i}(r)
$$

As mentioned above, this expression, as well as its range of applicability, has a theoretical foundation in the recently proposed $\mathrm{BFS}^{(1)}$ method. There is no formal derivation of this rule, but, in the framework of BFS, we can find an explanation for its origin and some idea about its validity and shortcomings. This will discussed at length elsewhere. Here, our goal is to provide three new approximate rules that would enable one to describe equilibrium alloy properties in terms of pure materials in a simple way. Although it is approximate, recent first-principles calculations closely follow the general behavior predicted by Eq. (1). Terakura et $\mathrm{al}^{(2)}$ analyzed the phase stability of several binary alloys systems composed of $\mathrm{Ni}, \mathrm{Pd}$ or $\mathrm{Pt}$ as one element and $\mathrm{Cu}, \mathrm{Ag}$ and $\mathrm{Au}$ as the other. From their LDA band calculations they found that their predictions for the excess energy $\Delta E_{m}(r)$ for the ordered 
alloy structures $A_{m} B_{4-m}(m=0, \ldots, 4)$ admit a simple parametrization of the form

$$
\Delta E_{m}(r)=\left(\frac{p_{n n}}{r}\right)^{2 n}-\left(\frac{q_{m}}{r}\right)^{n}+r_{m}
$$

where the parameters $p_{m}, q_{m}$ and $r_{m}$ are listed in Ref. 2 , with $n=3.5$ and $m=0,4$ represent the pure elements.. The excess energy for the disordered alloy can be represented by a similar functional form ${ }^{(3)}$,

$$
\Delta E_{D}(r, x)=\left(\frac{P_{x}}{r}\right)^{2 n}-\left(\frac{Q_{x}}{r}\right)^{n}+R_{x}
$$

with $P_{x}^{2 n}=\sum_{m=0}^{4} c_{m}(x) p_{m}^{2 n}, Q_{x}^{n}=\sum_{m=0}^{4} c_{m}(x) q_{m}^{n}$ and $R_{x}=\sum_{m=0}^{4} c_{m}(x) r_{m}$, where $c_{m}(x)=\left(\begin{array}{c}4 \\ m\end{array}\right) x^{m}(1-x)^{4-m}$. If these results behaved according to Eq. (1), then a direct consequence of this rule,

$$
\Delta E_{D}\left(r, x=\frac{m}{4}\right)=\Delta E_{m}(r) ; m=0, \ldots, 4
$$

would also have to be satisfied. In terms of the coefficients used in Ref. 2. this condition can be written as set of simultaneous requirements on these coefficients:

$$
P_{m / 4}^{2 n}=p_{m}^{n}, Q_{m / 4}^{n}=q_{m}^{n}, R_{m / 4}=r_{m} ; m=1,2,3
$$

We show the results of this comparison in Table 1 for the $\mathrm{Cu}-\mathrm{Ag}, \mathrm{Cu}-\mathrm{Au}$ and $\mathrm{Ag}-\mathrm{Au}$ alloys, where agreement in the two sides of Eq. (5) is a test of the sum rule. Although the comparison is not exact (it's not supposed to be), the agreement is surprisingly good, making the use of Eq. (1) a viable alternative to expressions ${ }^{(4)}$ for obtaining alloy cohesive energies, bulk moduli and lattice parameters, such as Vegard's law ${ }^{(5)}$ for lattice parameters.

The application of Eq. (1) is greatly simplified by the fact that the binding energy curve of a pure element $i, E_{i}(r)$, can be represented by a Rydberg function ${ }^{(6)}$

$$
E_{i}(r)=-E_{C, i}\left(1+a^{*}\right) e^{-a^{*}}
$$


with $a^{*}=q_{i}\left(r-a_{i}\right) / l_{i}$, where $a_{i}$ is the equilibrium lattice parameter of species $i$ and the scaling length $l_{i}$ is related to the cohesive energy of the pure element $E_{C, i}$ and its bulk modulus $B_{i}$ as $l_{i}^{2}=E_{C, i} / 12 \pi q_{i} B_{i} . \quad q_{i}$ is the ratio between the equilibrium Wigner-Seitz radius and equilibrium lattice parameter of species $i$.

Although it is a well-known fact that the binding energy curve (including alloys) is of a universal nature ${ }^{(6,7)}$, the functional form chosen to represent it is just one of many choices. If we used this functional form in Eq. (1), a representation of the alloys binding energy curve could only be achieved by a numerical fit to a Rydberg function. To avoid this complication, and in order to provide simple analytical expressions for approximate values of the static properties of the alloys, we expand the Rydberg function to second order on both sides of Eq. (1) thus obtaining a simple system of equations for $E_{C}(x), B(x)$ and $a(x)$. The ensuing expressions for these quantities are

$$
\begin{gathered}
a(x)=\frac{<Q a^{2} B>}{<Q a B>}, \\
B(x)=\frac{1}{Q_{x}} \frac{\left\langle Q a B>^{2}\right.}{\left\langle Q a^{2} B>\right.},
\end{gathered}
$$

and

$$
E(x)=<E>-<Q a^{3} B>+\frac{<Q a^{2} B>}{<Q a B>}
$$

where

$$
<O>=\sum_{i} x_{i} O_{i}
$$

and $Q_{i}=12 \pi q_{i}^{3}$.

If, for example, we focus our attention on binary alloys of elements with the same structure as the pure crystals, the $Q^{\prime} s$ cancel in Eqs.(7)-(9). Moreover, it is a simple exercise to examine these expressions for their departure from the corresponding average 
values $\Delta O(x)=\left\langle O_{x}\right\rangle-\langle O\rangle$ (for $O=a, B$ and $\left.E_{C}\right)$. The derivative at the origin of $\Delta O$ for each one of these quantities are

$$
\begin{gathered}
\left(\frac{d \Delta a(x)}{d x}\right)_{x_{A}=0}=\left(a_{A}-a_{B}\right) \frac{\left(a_{A} B_{A}-a_{B} B_{B}\right)}{a_{B} B_{B}}, \\
\left(\frac{d \Delta B(x)}{d x}\right)_{x_{A}=0}=-\frac{B_{A}}{a_{B}^{2}}\left(a_{A}-a_{B}\right)^{2}
\end{gathered}
$$

and

$$
\left(\frac{d \Delta E_{C}(x)}{d x}\right)_{x_{A}=0}=-a_{A} B_{A}\left(a_{A}-a_{B}\right)^{2}
$$

Similar expressions can be obtained for the limit $x_{A}=1$. Eq.(11) allows for deviations above and below the average values, whereas Eqs. (12) and (13) predict cohesive energies and bulk moduli below the average values for all concentrations. For this simple case, the maximum deviation of the lattice parameter from the average value $\langle a\rangle$ is given by

$$
\Delta a_{\max }=\frac{\delta \gamma \lambda}{(1+\gamma)\left(e_{A} \gamma+e_{B}\right)}
$$

where $\delta=a_{A}-a_{B}, \lambda=a_{A} e_{A}-a_{B} e_{B}, e_{i}=a_{i} B_{i}$ and $\gamma^{2}=e_{B} / e_{A}$. This maximum deviation occurs at $x_{A}=\frac{\gamma}{1+\gamma}$. Finally, it is straightforward to show that Vegard's law can be recovered as a particular case when $\gamma=1$.

The predictions of the trends predicted by Eq. (11) were successfully tested against 34 binary alloys of fcc $(\mathrm{Ag}, \mathrm{Al}, \mathrm{Au}, \mathrm{Cu}, \gamma$-Fe, Ir, Ni, Pd, Pt), bcc (Cr, Cs, $\alpha$-Fe, K, Li, Mo, $\mathrm{Na}, \mathrm{Nb}, \mathrm{Rb}, \mathrm{Ta}, \mathrm{V}, \mathrm{W}$ ) elements, as well as $\mathrm{Si}$ and Ge in their diamond and fcc phases for which complete experimental data of the solid solutions is available ${ }^{(8)}$. Similar results are found if other representations of the binding energy curves are used in our proposed rule (Eq. (1)).

The trends in Eq. (11) depend on a coupling between the lattice parameter and compressibility as might be expected intuitively. This result is a consequence of the fact that 
in our expression (Eq. (1)) the binding energy of the pure materials determine the behavior of the alloys. However, the specific functional form depends on the choice adopted for representing the binding energy curve (in this case, a simple quadratic expansion of the Rydberg function). The simple example studied in this work predicts the correct trends in the slopes (Eq. (11)) of the deviation from the average values as well as the trends in the relative deviations for different alloys.

We have presented a new method for estimating the cohesive energy, lattice parameter and bulk modulus for alloys from pure element properties. The method has been successfully tested by comparisons with ab-initio calculations and trends in experimental data. 


\section{References}

${ }^{1}$ Guillermo Bozzolo and John Ferrante, N.A.S.A. Technical Publication (1991) (in press); Guillermo Bozzolo, John Ferrante and John R. Smith (to be published)

${ }^{2}$ K. Terakura, T. Oguchi, T. Mohri and K. Watanabe, Phys. Rev. B35 2169 (1987); S. Takizawa, K. Terakura and T. Mohri, Phys. Rev. B39 5792 (1989)

${ }^{3}$ J. W. D. Connolly and A. R. Williams, Phys. Rev. B27 5169 (1983)

${ }^{4}$ G. Grimvall in Thermophysical Properties of Materials (North-Holland, 1986) and references therein

${ }^{5}$ L. Vegard, Z. Physik 517 (1921)

${ }^{6}$ J. H. Rose, J. R. Smith and J. Ferrante, Phys. Rev. B28 1835 (1983)

${ }^{7}$ J. H. Rose, J. R. Smith, F. Guinea and J. Ferrante, Phys. Rev. 292963 (1984)

${ }^{8}$ W. B. Pearson in Handbook of Lattice Spacings and Structures of Metals (Pergamon Press, 1967). 


\begin{tabular}{|l|l||c|c||c|c||c|c|}
\hline System & $\mathrm{m}$ & $\mathrm{P}_{m / 4}$ & $\mathrm{p}_{m}$ & $\mathrm{Q}_{m / 4}$ & $\mathrm{q}_{m}$ & $\mathrm{R}_{m / 4}$ & $\mathrm{r}_{m}$ \\
\hline $\mathrm{AgAu}_{3}$ & 1 & 8.46 & 8.45 & 11.37 & 11.35 & 1.98 & 1.96 \\
$\mathrm{AgAu}$ & 2 & 8.33 & 8.33 & 11.07 & 11.07 & 1.82 & 1.82 \\
$\mathrm{Ag}_{3} \mathrm{Au}$ & 3 & 8.21 & 8.21 & 10.77 & 10.77 & 1.66 & 1.66 \\
\hline $\mathrm{CuAg}_{3}$ & 1 & 7.84 & 7.82 & 10.11 & 10.08 & 1.51 & 1.50 \\
$\mathrm{CuAg}$ & 2 & 7.60 & 7.59 & 9.77 & 9.79 & 1.50 & 1.52 \\
$\mathrm{Cu}_{3} \mathrm{Ag}$ & 3 & 7.34 & 7.32 & 9.42 & 9.41 & 1.46 & 1.47 \\
\hline $\mathrm{CuAu}$ & 1 & 8.26 & 8.22 & 11.07 & 11.01 & 1.95 & 1.93 \\
$\mathrm{CuAu} \mathrm{Au}$ & 2 & 7.91 & 7.88 & 10.45 & 10.45 & 1.77 & 1.79 \\
$\mathrm{Cu}_{3} \mathrm{Au}$ & 3 & 7.52 & 7.47 & 9.78 & 9.75 & 1.59 & 1.59 \\
\hline
\end{tabular}

Table 1: Comparison of the parameters $(P, Q, R)$ and $(p, q, r)$ as defined by Eqs. (2) and (3) 
Public reporting burden for this collection of information is estimated to average 1 hour per response, including the time for reviewing instructions, searching existing data sources, gathering and maintaining the data needed, and completing and reviewing the collection of information. Send comments regarding this burden estimate or any other aspect of this collection of information, including suggestions for reducing this burden, to Washington Headquarters Services, Directorate for information Operations and Reports, 1215 Jefferson Davis Highway, Suite 1204, Arlington, VA 22202-4302, and to the Office of Management and Budget, Paperwork Reduction Project (0704-0188), Washington, DC 20503.
1. AGENCY USE ONLY (Leave blank)
2. REPORT DATE
October 1991
3. REPORT TYPE AND DATES COVERED
Technical Memorandum

4. TITLE AND SUBTITLE

A New Approximate Sum Rule for Bulk Alloy Properties

WU-505-90-51

Guillermo Bozzolo and John Ferrante

National Aeronautics and Space Administration

Lewis Research Center

5. FUNDING NUMBERS

Cleveland, Ohio 44135-3191

8. PERFORMING ORGANIZATION REPORT NUMBER

E-6606

9. SPONSORING/MONITORING AGENCY NAMES(S) AND ADDRESS(ES)

10. SPONSORING/MONITORING AGENCY REPORT NUMBER

National Aeronautics and Space Administration

Washington, D.C. 20546-0001

NASA TM-105282

\section{SUPPLEMENTARY NOTES}

Guillermo Bozzolo, Analex Corporation, 3001 Aerospace Parkway, Brook Park, Ohio 44142; John Ferrante, NASA Lewis Research Center. Responsible person, John Ferrante, (216) 433-6069.

12a. DISTRIBUTION/AVAILABILITY STATEMENT

12b. DISTRIBUTION CODE

Unclassified - Unlimited

Subject Category 26

13. ABSTRACT (Maximum 200 words)

We introduce a new, approximate sum rule for determining bulk properties of multicomponent systems, in terms of the pure components properties. We apply this expression for the study of lattice parameters, cohesive energies and bulk moduli of binary alloys. The correct experimental trends (i.e., departure from average values) are predicted in all cases.

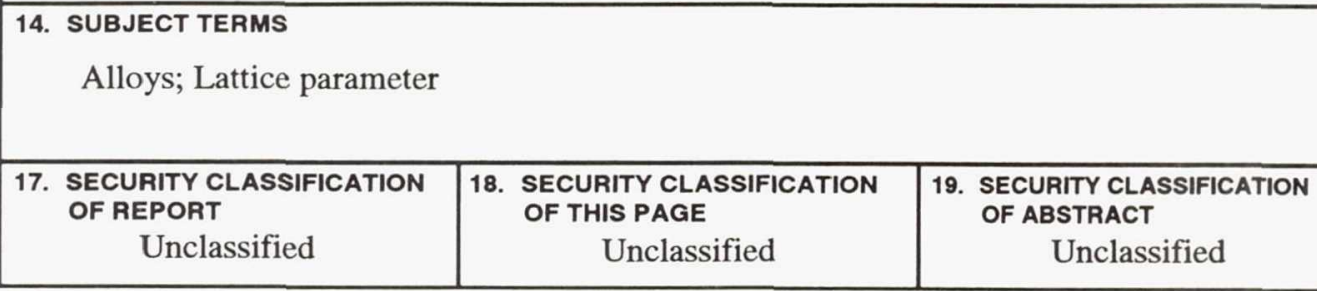

15. NUMBER OF PAGES

10

16. PRICE CODE

$\mathrm{A} 02$

20. LIMITATION OF ABSTRACT

